International Journal of Current Microbiology and Applied Sciences ISSN: 2319-7706 Volume 10 Number 10 (2021)

\title{
Effect of Storage Conditions on Seed Viability of China Aster [Callistephus chinensis (L.) Nees]
}

\author{
S. B. Vaidya ${ }^{1}$, P. B. Sable ${ }^{2^{*}}$ and V. B. Patil ${ }^{3}$ \\ ${ }^{1}$ Department of Horticulture, Mahatma Phule Krishi Vidyapeeth Rahuri, College of \\ Agriculture, Pune-411005, India \\ ${ }^{2}$ Department of Horticulture, Shri Shivaji Agriculture College, Amravati, 444602, \\ Maharashtra, India \\ ${ }^{3}$ Department of Plant Pathology, Late Shri Vasantrao Naik Marathwada Krishi Vidyapeeth, \\ Parbhani-431402, Maharashtra, India \\ *Corresponding author
}

Keywords

China aster, seed viability, seed storage, seed germination

Article Info

Accepted: 15 September 2021 Available Online: 10 October 2021
The study revealed that China aster seeds lose viability quickly if stored under ambient conditions. At the end of $12^{\text {th }}$ months, seeds stored at refrigerated condition $\left(5-7^{0} \mathrm{C}\right)$ still maintained high seed germination $(64.00 \%)$ at field condition well above the minimum seed certification standards. However, in case of ambient storage very drastic reduction in seed germination was observed. It was $32.43 \%$ for first months and reduced to $14.95 \%$ for second month by declining $53.90 \%$ as compared to first month and thereafter no germination was recorded at field condition. Results from the interaction effect indicated that previously stored seeds of China aster for six months in aluminium foil and kept under refrigerated condition recorded higher seed quality parameters viz. percent seed germination at field (92.67 and $72.00 \%)$, percent survival of seedlings (89.33 and 70.67\%), height of seedling (11.53 and $8.60 \mathrm{~cm})$, shoot length $(7.00$ and $5.10 \mathrm{~cm})$, root length $(4.53$ and $3.50 \mathrm{~cm})$, shoot weight $(0.42$ and $0.33 \mathrm{~g})$, root weight $(0.040$ and $0.027 \mathrm{~g})$, root:shoot ratio (0.095 and 0.082$)$ and percent seed germination at laboratory $(97.33$ and $76.00 \%$ ) for first and sixth month, respectively.

\section{Introduction}

China aster [Callistephus chinensis (L.) Nees.] a member of family Asteraceae, is one of the important commercial flower crop of our country. It is an important seed propagated annual flower crop mainly used for loose and cut flower production in Maharashtra, 
Karnataka, Tamil Nadu, Andhra Pradesh and West Bengal. The demand for quality seeds of this crop is continuous, as the crop is not season specific and grown throughout the year. However, the non-availability of quality seeds is one of the major problems in cultivation of this crop. The seeds of China aster are known to lose viability rapidly under ambient storage as reported by Salunkhe et al., 1987 and Yogeesha et al., 2004 and hence leading to poor emergence in the field. Seeds have remarkably complex and effective mechanism to remain viable or to ensure their long survival until next growing season. The present study was planned with the hypothesis that China aster seeds exhibit orthodox storage behaviour and lose viability rapidly under ambient storage. Suitable storage conditions prevent seed deterioration and thereby facilitate longer viability. Storage of seeds in moisture proof or moisture resistant package would be more valuable in prolonging seed germination and vigour.

To best of our knowledge, limited work has been reported regarding storage of China aster seeds. Therefore, this study was planned and executed with the objectives to investigate the proper method of storage of China aster seeds and to study the response of China aster seed to different packaging and storage conditions to maintain higher seed germination and viability for a longer period.

\section{Materials and Methods}

The present experiment was conducted in the Department of horticulture, College of Agriculture, Pune during 2013-14. The seeds of China aster were shade dried and stored for six months from April-October 2013 and thereafter at germination trials were undertaken at monthly intervals on field and laboratory conditions simultaneously from November 2013-April 2014. The field experiment was conducted in Factorial
Randomized Block Design (FRBD) consisting 14 treatment combinations with two factors as storage conditions viz. ambient and refrigerated $\left(5-7^{0} \mathrm{C}\right.$ and $90 \pm 2 \%$ R.H. $)$ storage and containers as second factor: tin box $\left(\mathrm{C}_{1}\right)$, glass bottle $\left(\mathrm{C}_{2}\right)$, polyethylene bag 200 gauge $\left(\mathrm{C}_{3}\right)$, polyethylene bag 400 gauge $\left(\mathrm{C}_{4}\right)$, vacuum packed polyethylene bag $\left(\mathrm{C}_{5}\right)$, muslin cloth bag $\left(\mathrm{C}_{6}\right)$ and aluminium foil $\left(\mathrm{C}_{7}\right)$. The observations were recorded on percent seed germination at field and laboratory conditions, percent survival of seedlings at field, height of seedling at time of transplanting, shoot length, root length, shoot weight, root weight and root:shoot ratio.

Seed germination at field level was recorded from every packaging in three replications of 100 seed each at monthly interval from November 2013-April 2014. The seedlings which were available for transplanting at 30 days after sowing were calculated for recording percent survival of seedlings at field. From the standard field germination test, ten normal seedlings were selected at random in each replication on final count. The shoot length was measured from collar region to the point of attachment of cotyledons and root length from the collar region to the tip of the primary root, sum of shoot and root length constitute the height of seedling and mean was calculated and expressed in centimetres. The shoot and root weight of 10 seedlings were measured and the rot: shoot ratio was also calculated. Germination percentage at laboratory level was determined as per ISTA rules for seed testing. The seeds were placed in rolled paper towels and hundred seeds of four replications were tested at a constant temperature of $25 \pm 2^{0} \mathrm{C}$ and $90 \%$ R.H. The number of normal seedlings was evaluated on 10th day and per cent germination was expressed on normal seedling basis (Anon, 2006). Factorial Completely Randomized Design (FCRD) was used for analysis of laboratory germination trials. 


\section{Results and Discussion}

\section{Effect of storage conditions (S)}

The observations revealed that seeds of China aster maintained significantly higher percent seed germination at field level (83.62 and $64.00 \%)$, seedling survival percentage (81.48 and $61.43 \%$ ), height of seedling (10.69 and $7.81 \mathrm{~cm})$, shoot length $(6.54$ and $4.60 \mathrm{~cm})$, root length $(4.15$ and $3.21 \mathrm{~cm})$, shoot weight $(0.38$ and $0.28 \mathrm{~g})$, root weight $(0.034$ and $0.021 \mathrm{~g})$, root:shoot ratio $(0.09$ and 0.07$)$ and percent seed germination at laboratory level (88.05 and $67.71 \%$ ) for first and sixth month, respectively after six months stored seeds of China aster as compared to those stored at ambient conditions (Table 1, 2, 3, 4, 5, 6, 7, 8 and 9).

Seeds stored at ambient conditions showed lower percent germination at field level (32.43 and $14.95 \%$ ) for first and second months, respectively. Drastic reduction in germination percentage of seeds stored at ambient storage at field condition was observed and it declined by $53.90 \%$ in second month as compared to first month and thereafter no germination was recorded for subsequent months. On the other hand such drastic reduction in germination percentage at laboratory condition was not observed and germination percentage varied from 38.14 to $1.57 \%$ for first and sixth month, respectively. Difference in germination percentage at laboratory and field condition may be due to the fact that seeds stored at low temperature but at a high relative humidity may lose viability rapidly when moved to a higher temperature. Gujar et al., (2001) also reported that the seeds of China aster stored under ambient conditions showed rapid decline in germination after seven months of storage in both moisture pervious and impervious containers. Similar findings were reported by Rao et al., (2003) where they observed a rapid decline in viability of marigold seeds under ambient conditions. These results of poor germination under ambient conditions are in conformity with Yogeesha et al., (2004) in China aster.

Under refrigerated storage, the loss of germination at slow rate could be due to reduced rate of metabolic activities and inactivation of enzymes required for retention of germination for longer period. Seeds stored under refrigerated condition showed seed germination at field level throughout six months. The temperature and moisture are the two major environmental factors that influence the seed longevity during storage. As per Harrington (1972) "rule of thumb" the seed looses half of their storage life with every $5^{\circ} \mathrm{C}$ increase in storage temperature between $0^{\circ} \mathrm{C}$ and $50^{\circ} \mathrm{C}$. Seedling vigour parameters like height of seedling, shoot length, root length, root weight, shoot weight, root:shoot ratio were found significant and decreased from first month to end of sixth month in all the containers. Similar findings were reported by Singh et al., (2004) where they observed gradual deterioration in seeds of African marigold with increase in storage period. All the seedling parameters were higher at refrigerated condition and lower at ambient storage in all the months of storage. Similar variation in seedling parameters influenced by storage conditions were reported in China aster by Yogeesha et al., (2004) and Gujar et al., (2001), in Matthiola by Dhatt et al., (2010), in snow flake by Cicek et al., (1994), in pasque flower by Sang et al., (1996).

\section{Effect of containers (C)}

Among the containers seeds of China aster stored in aluminium foil $\left(\mathrm{C}_{7}\right)$ showed significantly higher per cent seed germination at field level $(67.67$ and $36.00 \%)$, survival of seedlings percentage (64.33 and $35.33 \%$ ), height of seedling $(9.83$ and $4.30 \mathrm{~cm}$ ), shoot length $(6.10$ and $2.55 \mathrm{~cm})$, root length $(3.73$ 
and $1.75 \mathrm{~cm})$, shoot weight $(0.40$ and $0.16 \mathrm{~g})$, root weight $(0.037$ and $0.013 \mathrm{~g})$, root:shoot ratio (0.092 and 0.041) and per cent seed germination at laboratory level (73.67 and $43.50 \%$ ) followed by vacuum packed polyethylene bag $\left(\mathrm{C}_{5}\right)$ which showed percent seed germination at field level (64.17 and $34.83 \%)$, seedling survival percentage (61.33 and $33.67 \%$ ), height of seedling (9.65 and $4.17 \mathrm{~cm})$, shoot length $(6.03$ and $2.45 \mathrm{~cm})$, root length $(3.62$ and $1.72 \mathrm{~cm})$, shoot weight $(0.39$ and $0.15 \mathrm{~g})$, root weight $(0.036$ and $0.012 \mathrm{~g})$, root: shoot ratio (0.090 and 0.038$)$ and percent seed germination at laboratory level (70.50 and 36.67\%) for first and sixth month, respectively after six months stored seeds of China aster (Table 1, 2, 3, 4, 5, 6, 7, 8 and 9).

Seeds stored in muslin cloth bag $\left(\mathrm{C}_{6}\right)$ showed lowest per cent seed germination at field level (48.50 and 27.50\%), seedling survival percentage (45.17 and $25.17 \%$ ), height of seedling $(8.10$ and $3.50 \mathrm{~cm})$, shoot length (5.13 and $2.05 \mathrm{~cm})$, root length (2.97 and 1.45 $\mathrm{cm})$, shoot weight (0.34 and $0.12 \mathrm{~g})$, root weight (0.027 and $0.008 \mathrm{~g})$, root: shoot ratio (0.079 and 0.034) and percent seed germination at laboratory level (55.67 and $29.17 \%$ ) for first and sixth month, respectively.

The beneficial influence of moisture impervious containers (aluminium foil and vacuum packed polyethylene bag) noticed may be related to lesser decrease in seed vigour compared to those stored in relatively pervious containers like cloth bag. Similar findings were reported by Yogeesha et al., (2004) where they observed seeds of China aster stored in moisture resistant container of aluminium foil retained higher viability and vigour for longer period followed by moisture proof container and impervious containers.

Similarly, seedling vigour parameters like seedling length, shoot length, root length, root weight, shoot weight, root:shoot ratio of six months stored seeds of China aster were found to decrease from first month to end of sixth month in all the containers irrespective of storage conditions. This may be due to the phenomenon of ageing. Floris (1970) concluded that ageing is a progressive process accompanied by accumulation of toxic metabolites, which progressively depress germination and growth of seedlings with increased age.

Generally, seeds stored in moisture impervious containers like aluminium foil, vacuum packed polyethylene bag, tin box, glass bottle, polyethylene bag (200 gauge) and polyethylene bag (400 gauge) maintained viability and vigour for longer period compared to those stored in moisture pervious container like cloth bag. This beneficial influence is mainly attributed to lesser moisture fluctuation in seeds stored in moisture impervious containers. In sealed containers viz. glass bottle and tin box the seed vigour characters were better than the moisture impervious container such as muslin cloth bag, this may be due to completely closed system in the containers where $\mathrm{CO}_{2}$ replaces $\mathrm{O}_{2}$ and this accumulation of $\mathrm{CO}_{2}$ inhibits respiration thus paving way for extending seed longevity. The quality of vacuum packed polyethylene bag may be due to stoppage of respiration of seeds inside the container as all the air is removed out during vacuum packaging, thus all the metabolic activities come to rest paving way for longer storage (Justice and Bass, 1978).

In aluminium foil higher percent seed germination and seedling vigour parameters can be attributed to non-permeability of moisture. Aluminium foil has a low moisture vapour transmission rate and the thick films transmit almost no water vapour at all. (Justice and Bass, 1978). The superiority of aluminium 
foil in maintaining viability was also reported by Yogeesha (2004), Gnyandev (2006) and Deepa et al., (2008) in China aster, Bharathi (2002), Dhatt and Kumar (2010a) in gaillardia, Poonam (2002) in Zinnia, Selvaraju and
Selvaraj (1999) in marigold, Dubey et al., (2002) in cosmos, Dhatt and Kumar (2009) in daisy, Dhatt and Kumar (2010c) in Coreopsis lanceolata

Table.1 Effect of storage conditions and containers on germination percentage at field condition after six months of stored China aster seeds.

\begin{tabular}{|c|c|c|c|c|c|c|}
\hline Treatments & $1^{\text {st }}$ month & $2^{\text {nd }}$ month & $3^{\text {rd }}$ month & $4^{\text {th }}$ month & $5^{\text {th }}$ month & $6^{\text {th }}$ month \\
\hline \multicolumn{7}{|c|}{ Storage conditions (S) } \\
\hline Ambient $\left(\mathbf{S}_{1}\right)$ & $\begin{array}{c}32.43 \\
(34.56)^{*}\end{array}$ & $\begin{array}{c}14.95 \\
(22.47)\end{array}$ & $\begin{array}{c}0.00 \\
(0.00)\end{array}$ & $\begin{array}{c}0.00 \\
(0.00)\end{array}$ & $\begin{array}{c}0.00 \\
(0.00)\end{array}$ & $\begin{array}{c}0.00 \\
(0.00)\end{array}$ \\
\hline Refrigerated $\left(\mathbf{S}_{2}\right)$ & $\begin{array}{c}83.62 \\
(66.59)\end{array}$ & $\begin{array}{c}79.52 \\
(63.39)\end{array}$ & $\begin{array}{c}76.43 \\
(61.18)\end{array}$ & $\begin{array}{c}72.86 \\
(58.79)\end{array}$ & $\begin{array}{c}69.48 \\
(56.59)\end{array}$ & $\begin{array}{c}64.00 \\
(53.20)\end{array}$ \\
\hline S.E. \pm & 0.53 & 0.58 & 0.46 & 0.48 & 0.48 & 0.31 \\
\hline C.D.@ 5\% & 1.53 & 1.68 & 1.33 & 1.41 & 1.39 & 0.89 \\
\hline \multicolumn{7}{|c|}{ Storage Containers (C) } \\
\hline Tin box $\left(C_{1}\right)$ & $\begin{array}{c}52.00 \\
(46.33)\end{array}$ & $\begin{array}{c}42.00 \\
(38.99)\end{array}$ & $\begin{array}{c}35.17 \\
(28.52)\end{array}$ & $\begin{array}{c}33.67 \\
(27.58)\end{array}$ & $\begin{array}{c}31.50 \\
(26.27)\end{array}$ & $\begin{array}{c}29.33 \\
(25.00)\end{array}$ \\
\hline Glass bottle $\left(C_{2}\right)$ & $\begin{array}{c}54.83 \\
(48.23)\end{array}$ & $\begin{array}{c}44.50 \\
(40.83)\end{array}$ & $\begin{array}{c}36.83 \\
(29.58)\end{array}$ & $\begin{array}{c}35.17 \\
(28.52)\end{array}$ & $\begin{array}{c}33.67 \\
(27.581)\end{array}$ & $\begin{array}{c}30.83 \\
(25.88)\end{array}$ \\
\hline Polyethylene bag 200 gauge $\left(C_{3}\right)$ & $\begin{array}{c}58.00 \\
(50.40)\end{array}$ & $\begin{array}{c}47.50 \\
(43.09)\end{array}$ & $\begin{array}{c}38.67 \\
(30.83)\end{array}$ & $\begin{array}{c}36.50 \\
(29.37)\end{array}$ & $\begin{array}{c}35.33 \\
(28.66)\end{array}$ & $\begin{array}{c}32.33 \\
(26.78)\end{array}$ \\
\hline Polyethylene bag 400 gauge $\left(C_{4}\right)$ & $\begin{array}{c}61.00 \\
(52.56)\end{array}$ & $\begin{array}{c}50.17 \\
(45.17)\end{array}$ & $\begin{array}{c}39.83 \\
(31.61)\end{array}$ & $\begin{array}{c}37.50 \\
(30.02)\end{array}$ & $\begin{array}{c}36.00 \\
(29.06)\end{array}$ & $\begin{array}{c}33.17 \\
(27.28)\end{array}$ \\
\hline Vaccum packed polyethylene bag $\left(C_{5}\right)$ & $\begin{array}{c}64.17 \\
(54.84)\end{array}$ & $\begin{array}{c}52.33 \\
(46.81)\end{array}$ & $\begin{array}{c}40.83 \\
(32.36)\end{array}$ & $\begin{array}{c}39.17 \\
(31.17)\end{array}$ & $\begin{array}{c}37.00 \\
(29.68)\end{array}$ & $\begin{array}{c}34.83 \\
(28.29)\end{array}$ \\
\hline Muslin cloth $\left(\mathbf{C}_{6}\right)$ & $\begin{array}{c}48.50 \\
(43.98)\end{array}$ & $\begin{array}{c}39.67 \\
(37.14)\end{array}$ & $\begin{array}{c}34.00 \\
(27.78)\end{array}$ & $\begin{array}{c}32.33 \\
(26.78)\end{array}$ & $\begin{array}{c}31.00 \\
(25.98)\end{array}$ & $\begin{array}{c}27.50 \\
(23.94)\end{array}$ \\
\hline Aluminium foil $\left(\mathbf{C}_{7}\right)$ & $\begin{array}{c}67.67 \\
(57.68)\end{array}$ & $\begin{array}{c}54.50 \\
(48.45)\end{array}$ & $\begin{array}{c}42.17 \\
(33.41)\end{array}$ & $\begin{array}{c}40.67 \\
(32.29)\end{array}$ & $\begin{array}{c}38.67 \\
(30.84)\end{array}$ & $\begin{array}{c}36.00 \\
(29.04)\end{array}$ \\
\hline S.E. \pm & 0.40 & 0.44 & 0.35 & 0.37 & 0.37 & 0.23 \\
\hline C.D.@5\% & 1.17 & 1.28 & 1.01 & 1.07 & 1.06 & 0.68 \\
\hline \multicolumn{7}{|c|}{ Interaction effect(SxC) } \\
\hline $\mathbf{S}_{1} \mathbf{C}_{1}$ & $\begin{array}{c}25.67 \\
(30.37)\end{array}$ & $\begin{array}{c}10.67 \\
(19.03)\end{array}$ & $\begin{array}{c}0.00 \\
(0.00)\end{array}$ & $\begin{array}{c}0.00 \\
(0.00)\end{array}$ & $\begin{array}{c}0.00 \\
(0.00)\end{array}$ & $\begin{array}{c}0.00 \\
(0.00)\end{array}$ \\
\hline $\mathbf{S}_{1} \mathbf{C}_{2}$ & $\begin{array}{c}29.33 \\
(32.76)\end{array}$ & $\begin{array}{c}12.33 \\
(20.46)\end{array}$ & $\begin{array}{c}0.00 \\
(0.00)\end{array}$ & $\begin{array}{c}0.00 \\
(0.00)\end{array}$ & $\begin{array}{c}0.00 \\
(0.00)\end{array}$ & $\begin{array}{c}0.00 \\
(0.00)\end{array}$ \\
\hline $\mathbf{S}_{1} \mathbf{C}_{3}$ & $\begin{array}{c}33.33 \\
(35.26)\end{array}$ & $\begin{array}{c}15.33 \\
(22.93)\end{array}$ & $\begin{array}{c}0.00 \\
(0.00)\end{array}$ & $\begin{array}{c}0.00 \\
(0.00)\end{array}$ & $\begin{array}{c}0.00 \\
(0.00)\end{array}$ & $\begin{array}{c}0.00 \\
(0.00)\end{array}$ \\
\hline $\mathbf{S}_{1} \mathbf{C}_{4}$ & $\begin{array}{c}35.67 \\
(36.67)\end{array}$ & $\begin{array}{c}16.67 \\
(24.08)\end{array}$ & $\begin{array}{c}0.00 \\
(0.00)\end{array}$ & $\begin{array}{c}0.00 \\
(0.00)\end{array}$ & $\begin{array}{c}0.00 \\
(0.00)\end{array}$ & $\begin{array}{c}0.00 \\
(0.00)\end{array}$ \\
\hline $\mathrm{S}_{1} \mathrm{C}_{5}$ & 39.00 & 19.33 & 0.00 & 0.00 & 0.00 & 0.00 \\
\hline
\end{tabular}




\begin{tabular}{|c|c|c|c|c|c|c|}
\hline & $(38.62)$ & $(26.06)$ & $(0.00)$ & $(0.00)$ & $(0.00)$ & $(0.00)$ \\
\hline $\mathbf{S}_{\mathbf{1}} \mathbf{C}_{\mathbf{6}}$ & 21.33 & 8.67 & 0.00 & 0.00 & 0.00 & 0.00 \\
& $(27.48)$ & $(17.00)$ & $(0.00)$ & $(0.00)$ & $(0.00)$ & $(0.00)$ \\
\hline $\mathbf{S}_{\mathbf{1}} \mathbf{C}_{\mathbf{7}}$ & 42.67 & 21.67 & 0.00 & 0.00 & 0.00 & 0.00 \\
& $(40.78)$ & $(27.69)$ & $(0.00)$ & $(0.00)$ & $(0.00)$ & $(0.00)$ \\
\hline $\mathbf{S}_{\mathbf{2}} \mathbf{C}_{\mathbf{1}}$ & 78.33 & 73.33 & 70.33 & 67.33 & 63.00 & 58.67 \\
& $(62.29)$ & $(58.94)$ & $(57.05)$ & $(55.17)$ & $(52.54)$ & $(50.00)$ \\
\hline $\mathbf{S}_{\mathbf{2}} \mathbf{C}_{\mathbf{2}}$ & 80.33 & 76.67 & 73.67 & 70.33 & 67.33 & 61.67 \\
& $(63.69)$ & $(61.20)$ & $(59.17)$ & $(57.05)$ & $(55.16)$ & $(51.75)$ \\
\hline $\mathbf{S}_{\mathbf{2}} \mathbf{C}_{\mathbf{3}}$ & 82.67 & 79.67 & 77.33 & 73.00 & 70.67 & 64.67 \\
& $(65.55)$ & $(63.25)$ & $(61.66)$ & $(58.75)$ & $(57.33)$ & $(53.55)$ \\
\hline $\mathbf{S}_{\mathbf{2}} \mathbf{C}_{\mathbf{4}}$ & 86.33 & 83.67 & 79.67 & 75.00 & 72.00 & 66.33 \\
& $(68.44)$ & $(66.27)$ & $(63.22)$ & $(60.05)$ & $(58.12)$ & $(54.56)$ \\
\hline $\mathbf{S}_{\mathbf{2}} \mathbf{C}_{\mathbf{5}}$ & 89.33 & 85.33 & 81.67 & 78.33 & 74.00 & 69.67 \\
& $(71.05)$ & $(67.55)$ & $(64.73)$ & $(62.33)$ & $(59.37)$ & $(56.59)$ \\
\hline $\mathbf{S}_{\mathbf{2}} \mathbf{C}_{\mathbf{6}}$ & 75.67 & 70.67 & 68.00 & 64.67 & 62.00 & 55.00 \\
& $(60.48)$ & $(57.28)$ & $(55.57)$ & $(53.57)$ & $(51.95)$ & $(47.87)$ \\
\hline $\mathbf{S}_{\mathbf{2}} \mathbf{C}_{\mathbf{7}}$ & 92.67 & 87.33 & 84.33 & 81.33 & 77.33 & 72.00 \\
& $(74.58)$ & $(69.21)$ & $(66.83)$ & $(64.59)$ & $(61.68)$ & $(58.07)$ \\
\hline $\mathbf{S . E . \pm}_{\mathbf{C} . D .} @ \mathbf{5 \%}$ & 1.40 & 1.53 & 1.21 & 1.28 & 1.27 & 0.81 \\
\hline S. & $\mathrm{NS}$ & $\mathrm{NS}$ & 3.51 & 3.72 & 3.69 & 2.36 \\
\hline
\end{tabular}

NS-Non Significant

*Figures in parenthesis indicate arcsine transformed values

Table.2 Effect of storage conditions and containers on survival of seedling percentage at field condition after six months of stored China aster seeds.

\begin{tabular}{|c|c|c|c|c|c|c|}
\hline Treatment & $\begin{array}{c}1^{\text {st }} \\
\text { month }\end{array}$ & $\begin{array}{c}2^{\text {nd }} \\
\text { month }\end{array}$ & $\begin{array}{c}3^{\text {rd }} \\
\text { month }\end{array}$ & $\begin{array}{c}4^{\text {th }} \\
\text { month }\end{array}$ & $\begin{array}{c}5^{\text {th }} \\
\text { month }\end{array}$ & $\begin{array}{c}6^{\text {th }} \\
\text { month }\end{array}$ \\
\hline \multicolumn{7}{|c|}{ Storage conditions (S) } \\
\hline Ambient $\left(\mathbf{S}_{1}\right)$ & $\begin{array}{c}28.38 \\
(31.92) \\
*\end{array}$ & $\begin{array}{l}10.10 \\
(18.27)\end{array}$ & $\begin{array}{c}0.00 \\
(0.00)\end{array}$ & $\begin{array}{c}0.00 \\
(0.00)\end{array}$ & $\begin{array}{c}0.00 \\
(0.00)\end{array}$ & $\begin{array}{c}0.00 \\
(0.00)\end{array}$ \\
\hline Refrigerated $\left(\mathbf{S}_{2}\right)$ & $\begin{array}{c}81.48 \\
(64.81)\end{array}$ & $\begin{array}{c}77.29 \\
(61.69)\end{array}$ & $\begin{array}{c}72.90 \\
(58.80)\end{array}$ & $\begin{array}{c}69.05 \\
(56.31)\end{array}$ & $\begin{array}{c}65.24 \\
(53.96)\end{array}$ & $\begin{array}{c}61.43 \\
(51.68)\end{array}$ \\
\hline S.E. \pm & 0.60 & 0.54 & 0.39 & 0.37 & 0.39 & 0.32 \\
\hline C.D.@ 5\% & 1.76 & 1.58 & 1.13 & 1.07 & 1.13 & 0.93 \\
\hline \multicolumn{7}{|c|}{ Storage Containers (C) } \\
\hline Tin box $\left(C_{1}\right)$ & $\begin{array}{c}49.17 \\
(44.44)\end{array}$ & $\begin{array}{c}40.50 \\
(37.49)\end{array}$ & $\begin{array}{c}33.67 \\
(27.58)\end{array}$ & $\begin{array}{c}32.17 \\
(26.67)\end{array}$ & $\begin{array}{c}30.50 \\
(25.68)\end{array}$ & $\begin{array}{c}27.67 \\
(24.03)\end{array}$ \\
\hline Glass bottle $\left(C_{2}\right)$ & $\begin{array}{c}51.83 \\
(46.26)\end{array}$ & $\begin{array}{c}41.83 \\
(38.42)\end{array}$ & $\begin{array}{c}35.00 \\
(28.40)\end{array}$ & $\begin{array}{c}32.67 \\
(26.98)\end{array}$ & $\begin{array}{c}31.83 \\
(26.47)\end{array}$ & $\begin{array}{c}29.67 \\
(25.20)\end{array}$ \\
\hline $\begin{array}{l}\text { Polyethylene bag } 200 \text { gauge } \\
\left(C_{3}\right)\end{array}$ & $\begin{array}{c}55.17 \\
(48.47)\end{array}$ & $\begin{array}{c}44.00 \\
(40.31)\end{array}$ & $\begin{array}{c}36.33 \\
(29.25)\end{array}$ & $\begin{array}{c}34.17 \\
(27.90)\end{array}$ & $\begin{array}{c}32.67 \\
(26.98)\end{array}$ & $\begin{array}{c}31.33 \\
(26.17)\end{array}$ \\
\hline Polyethylene bag 400 gauge & 57.50 & 44.50 & 37.83 & 36.17 & 34.17 & 32.17 \\
\hline
\end{tabular}




\begin{tabular}{|c|c|c|c|c|c|c|}
\hline$\left(\mathbf{C}_{4}\right)$ & $(50.06)$ & $(40.72)$ & $(30.25)$ & (29.15) & $(27.90)$ & $(26.67)$ \\
\hline $\begin{array}{c}\text { Vaccum packed polyethylene } \\
\text { bag }\left(\mathbf{C}_{5}\right)\end{array}$ & $\begin{array}{c}61.33 \\
(52.91)\end{array}$ & $\begin{array}{c}46.83 \\
(42.52)\end{array}$ & $\begin{array}{c}39.00 \\
(31.04)\end{array}$ & $\begin{array}{c}37.33 \\
(29.90)\end{array}$ & $\begin{array}{l}35.00 \\
(28.40)\end{array}$ & $\begin{array}{c}33.67 \\
(27.58)\end{array}$ \\
\hline Muslin cloth $\left(\mathbf{C}_{6}\right)$ & $\begin{array}{c}45.17 \\
(41.47)\end{array}$ & $\begin{array}{c}38.67 \\
(35.77)\end{array}$ & $\begin{array}{c}32.50 \\
(26.88)\end{array}$ & $\begin{array}{l}30.50 \\
(25.68)\end{array}$ & $\begin{array}{l}27.67 \\
(24.03)\end{array}$ & $\begin{array}{c}25.17 \\
(22.60)\end{array}$ \\
\hline Aluminium foil $\left(\mathbf{C}_{7}\right)$ & $\begin{array}{c}64.33 \\
(54.95)\end{array}$ & $\begin{array}{c}49.50 \\
(44.61)\end{array}$ & $\begin{array}{c}40.83 \\
(32.38)\end{array}$ & $\begin{array}{c}38.67 \\
(30.80)\end{array}$ & $\begin{array}{c}36.50 \\
(29.37)\end{array}$ & $\begin{array}{c}35.33 \\
(28.62)\end{array}$ \\
\hline S.E. \pm & 0.46 & 0.41 & 0.30 & 0.28 & 0.30 & 0.24 \\
\hline C.D.@ 5\% & 1.34 & 1.20 & 0.86 & 0.81 & 0.86 & 0.71 \\
\hline \multicolumn{7}{|c|}{ Interaction effect (SxC) } \\
\hline$S_{1} C_{1}$ & $\begin{array}{c}21.67 \\
(27.71)\end{array}$ & $\begin{array}{c}7.67 \\
(16.02)\end{array}$ & $\begin{array}{c}0.00 \\
(0.00)\end{array}$ & $\begin{array}{c}0.00 \\
(0.00)\end{array}$ & $\begin{array}{c}0.00 \\
(0.00)\end{array}$ & $\begin{array}{c}0.00 \\
(0.00)\end{array}$ \\
\hline $\mathbf{S}_{1} \mathbf{C}_{2}$ & $\begin{array}{c}25.00 \\
(29.96)\end{array}$ & $\begin{array}{c}8.00 \\
(16.35)\end{array}$ & $\begin{array}{c}0.00 \\
(0.00)\end{array}$ & $\begin{array}{c}0.00 \\
(0.00)\end{array}$ & $\begin{array}{c}0.00 \\
(0.00)\end{array}$ & $\begin{array}{c}0.00 \\
(0.00)\end{array}$ \\
\hline $\mathrm{S}_{1} \mathrm{C}_{3}$ & $\begin{array}{c}30.33 \\
(33.41)\end{array}$ & $\begin{array}{c}10.67 \\
(19.03)\end{array}$ & $\begin{array}{c}0.00 \\
(0.00)\end{array}$ & $\begin{array}{c}0.00 \\
(0.00)\end{array}$ & $\begin{array}{c}0.00 \\
(0.00)\end{array}$ & $\begin{array}{c}0.00 \\
(0.00)\end{array}$ \\
\hline $\mathrm{S}_{1} \mathrm{C}_{4}$ & $\begin{array}{c}32.33 \\
(34.64)\end{array}$ & $\begin{array}{c}11.00 \\
(19.36)\end{array}$ & $\begin{array}{c}0.00 \\
(0.00)\end{array}$ & $\begin{array}{c}0.00 \\
(0.00)\end{array}$ & $\begin{array}{c}0.00 \\
(0.00)\end{array}$ & $\begin{array}{c}0.00 \\
(0.00)\end{array}$ \\
\hline$S_{1} C_{5}$ & $\begin{array}{c}35.33 \\
(36.45)\end{array}$ & $\begin{array}{c}12.67 \\
(20.81)\end{array}$ & $\begin{array}{c}0.00 \\
(0.00)\end{array}$ & $\begin{array}{c}0.00 \\
(0.00)\end{array}$ & $\begin{array}{c}0.00 \\
(0.00)\end{array}$ & $\begin{array}{c}0.00 \\
(0.00)\end{array}$ \\
\hline$S_{1} C_{6}$ & $\begin{array}{c}14.67 \\
(22.42)\end{array}$ & $\begin{array}{c}6.00 \\
(13.87)\end{array}$ & $\begin{array}{c}0.00 \\
(0.00)\end{array}$ & $\begin{array}{c}0.00 \\
(0.00)\end{array}$ & $\begin{array}{c}0.00 \\
(0.00)\end{array}$ & $\begin{array}{c}0.00 \\
(0.00)\end{array}$ \\
\hline $\mathbf{S}_{1} \mathbf{C}_{7}$ & $\begin{array}{c}39.33 \\
(38.81)\end{array}$ & $\begin{array}{c}14.67 \\
(22.47)\end{array}$ & $\begin{array}{c}0.00 \\
(0.00)\end{array}$ & $\begin{array}{c}0.00 \\
(0.00)\end{array}$ & $\begin{array}{c}0.00 \\
(0.00)\end{array}$ & $\begin{array}{c}0.00 \\
(0.00)\end{array}$ \\
\hline $\mathbf{S}_{2} \mathbf{C}_{1}$ & $\begin{array}{c}76.67 \\
(61.18)\end{array}$ & $\begin{array}{c}73.33 \\
(58.96)\end{array}$ & $\begin{array}{c}67.33 \\
(55.16)\end{array}$ & $\begin{array}{c}64.33 \\
(53.34)\end{array}$ & $\begin{array}{c}61.00 \\
(51.36)\end{array}$ & $\begin{array}{c}55.33 \\
(48.07)\end{array}$ \\
\hline $\mathbf{S}_{2} \mathbf{C}_{2}$ & $\begin{array}{c}78.67 \\
(62.56)\end{array}$ & $\begin{array}{c}75.67 \\
(60.49)\end{array}$ & $\begin{array}{c}70.00 \\
(56.81)\end{array}$ & $\begin{array}{c}65.33 \\
(53.96)\end{array}$ & $\begin{array}{c}63.67 \\
(52.94)\end{array}$ & $\begin{array}{c}59.33 \\
(50.39)\end{array}$ \\
\hline $\mathbf{S}_{2} \mathbf{C}_{3}$ & $\begin{array}{c}80.00 \\
(63.53)\end{array}$ & $\begin{array}{c}77.33 \\
(61.59)\end{array}$ & $\begin{array}{c}72.67 \\
(58.50)\end{array}$ & $\begin{array}{c}68.33 \\
(55.80)\end{array}$ & $\begin{array}{c}65.33 \\
(53.96)\end{array}$ & $\begin{array}{c}62.67 \\
(52.34)\end{array}$ \\
\hline $\mathbf{S}_{2} \mathbf{C}_{4}$ & $\begin{array}{c}82.67 \\
(65.47)\end{array}$ & $\begin{array}{c}78.00 \\
(62.09)\end{array}$ & $\begin{array}{c}75.67 \\
(60.51)\end{array}$ & $\begin{array}{c}72.33 \\
(58.30)\end{array}$ & $\begin{array}{c}68.33 \\
(55.80)\end{array}$ & $\begin{array}{c}64.33 \\
(53.34)\end{array}$ \\
\hline $\mathbf{S}_{2} \mathbf{C}_{5}$ & $\begin{array}{c}87.33 \\
(69.36)\end{array}$ & $\begin{array}{c}81.00 \\
(64.23)\end{array}$ & $\begin{array}{c}78.00 \\
(62.08)\end{array}$ & $\begin{array}{c}74.67 \\
(59.81)\end{array}$ & $\begin{array}{c}70.00 \\
(56.81)\end{array}$ & $\begin{array}{c}67.33 \\
(55.16)\end{array}$ \\
\hline $\mathbf{S}_{2} \mathbf{C}_{6}$ & $\begin{array}{c}75.67 \\
(60.52)\end{array}$ & $\begin{array}{c}71.33 \\
(57.67)\end{array}$ & $\begin{array}{c}65.00 \\
(53.76)\end{array}$ & $\begin{array}{c}61.00 \\
(51.36)\end{array}$ & $\begin{array}{c}55.33 \\
(48.07)\end{array}$ & $\begin{array}{c}50.33 \\
(45.19)\end{array}$ \\
\hline $\mathbf{S}_{2} \mathbf{C}_{7}$ & $\begin{array}{c}89.33 \\
(71.09)\end{array}$ & $\begin{array}{c}84.33 \\
(66.76)\end{array}$ & $\begin{array}{c}81.67 \\
(64.76)\end{array}$ & $\begin{array}{c}77.33 \\
(61.60)\end{array}$ & $\begin{array}{c}73.00 \\
(58.74)\end{array}$ & $\begin{array}{c}70.67 \\
(57.24)\end{array}$ \\
\hline S.E. \pm & 1.60 & 1.44 & 1.03 & 0.97 & 1.03 & 0.84 \\
\hline C.D. @ 5\% & NS & NS & 2.99 & 2.82 & 2.98 & 2.45 \\
\hline
\end{tabular}


Table.3 Effect of storage conditions and containers on height of seedlings $(\mathrm{cm})$ at transplanting after six months of stored China aster seeds.

\begin{tabular}{|c|c|c|c|c|c|c|}
\hline Treatments & $\begin{array}{c}1^{\text {st }} \\
\text { month }\end{array}$ & $\begin{array}{c}2^{\text {nd }} \\
\text { month }\end{array}$ & $\begin{array}{c}3^{\text {rd }} \\
\text { month }\end{array}$ & $\begin{array}{c}4^{\text {th }} \\
\text { month }\end{array}$ & $\begin{array}{c}5^{\text {th }} \\
\text { month }\end{array}$ & $\begin{array}{c}6^{\text {th }} \\
\text { month }\end{array}$ \\
\hline \multicolumn{7}{|c|}{ Storage conditions (S) } \\
\hline Ambient $\left(\mathbf{S}_{1}\right)$ & 7.52 & 7.02 & 0.00 & 0.00 & 0.00 & 0.00 \\
\hline Refrigerated $\left(\mathbf{S}_{2}\right)$ & 10.69 & 9.37 & 8.88 & 8.40 & 8.02 & 7.81 \\
\hline S.E. \pm & 0.07 & 0.09 & 0.05 & 0.07 & 0.06 & 0.07 \\
\hline C.D.@ 5\% & 0.22 & 0.25 & 0.16 & 0.22 & 0.18 & 0.19 \\
\hline \multicolumn{7}{|c|}{ Storage Containers (C) } \\
\hline Tin box $\left(C_{1}\right)$ & 8.52 & 7.53 & 4.27 & 3.98 & 3.78 & 3.65 \\
\hline Glass bottle $\left(C_{2}\right)$ & 8.97 & 7.93 & 4.32 & 4.10 & 3.90 & 3.83 \\
\hline $\begin{array}{c}\text { Polyethylene bag } 200 \text { gauge } \\
\left(C_{3}\right)\end{array}$ & 9.22 & 8.12 & 4.43 & 4.22 & 3.98 & 3.90 \\
\hline $\begin{array}{l}\text { Polyethylene bag } 400 \text { gauge } \\
\left(\mathrm{C}_{4}\right)\end{array}$ & 9.47 & 8.38 & 4.50 & 4.30 & 4.10 & 4.00 \\
\hline $\begin{array}{c}\text { Vaccum packed polyethylene } \\
\text { bag }\left(\mathbf{C}_{5}\right)\end{array}$ & 9.65 & 8.88 & 4.65 & 4.40 & 4.25 & 4.17 \\
\hline Muslin cloth $\left(\mathbf{C}_{6}\right)$ & 8.10 & 7.30 & 4.10 & 3.85 & 3.60 & 3.50 \\
\hline Aluminium foil $\left(\mathrm{C}_{7}\right)$ & 9.83 & 9.23 & 4.82 & 4.55 & 4.45 & 4.30 \\
\hline S.E. \pm & 0.06 & 0.07 & 0.04 & 0.06 & 0.05 & 0.05 \\
\hline C.D.@ 5\% & 0.16 & 0.19 & 0.12 & 0.17 & 0.13 & 0.15 \\
\hline \multicolumn{7}{|c|}{ Interaction effect(SxC) } \\
\hline$S_{1} C_{1}$ & 7.07 & 6.17 & 0.00 & 0.00 & 0.00 & 0.00 \\
\hline$S_{1} C_{2}$ & 7.30 & 6.80 & 0.00 & 0.00 & 0.00 & 0.00 \\
\hline $\mathrm{S}_{1} \mathrm{C}_{3}$ & 7.57 & 7.10 & 0.00 & 0.00 & 0.00 & 0.00 \\
\hline $\mathrm{S}_{1} \mathbf{C}_{4}$ & 7.80 & 7.30 & 0.00 & 0.00 & 0.00 & 0.00 \\
\hline $\mathrm{S}_{1} \mathrm{C}_{5}$ & 8.00 & 7.83 & 0.00 & 0.00 & 0.00 & 0.00 \\
\hline$S_{1} C_{6}$ & 6.80 & 6.00 & 0.00 & 0.00 & 0.00 & 0.00 \\
\hline $\mathbf{S}_{1} \mathbf{C}_{7}$ & 8.13 & 7.97 & 0.00 & 0.00 & 0.00 & 0.00 \\
\hline $\mathbf{S}_{2} \mathbf{C}_{1}$ & 9.97 & 8.90 & 8.53 & 7.97 & 7.57 & 7.30 \\
\hline $\mathbf{S}_{2} \mathbf{C}_{2}$ & 10.63 & 9.07 & 8.63 & 8.20 & 7.80 & 7.67 \\
\hline $\mathbf{S}_{2} \mathbf{C}_{3}$ & 10.87 & 9.13 & 8.87 & 8.43 & 7.97 & 7.80 \\
\hline $\mathrm{S}_{2} \mathrm{C}_{4}$ & 11.13 & 9.47 & 9.00 & 8.60 & 8.20 & 8.00 \\
\hline $\mathbf{S}_{2} \mathbf{C}_{5}$ & 11.30 & 9.93 & 9.30 & 8.80 & 8.50 & 8.33 \\
\hline$S_{2} C_{6}$ & 9.40 & 8.60 & 8.20 & 7.70 & 7.20 & 7.00 \\
\hline $\mathbf{S}_{2} \mathbf{C}_{7}$ & 11.53 & 10.50 & 9.63 & 9.10 & 8.90 & 8.60 \\
\hline S.E. \pm & 0.20 & 0.23 & 0.14 & 0.20 & 0.16 & 0.18 \\
\hline C.D. @ 5\% & NS & NS & 0.41 & 0.57 & 0.47 & 0.51 \\
\hline
\end{tabular}

NS-Non Significant 
Table.4 Effect of storage conditions and containers on shoot length of seedlings $(\mathrm{cm})$ at transplanting after six months of stored China aster seeds.

\begin{tabular}{|c|c|c|c|c|c|c|}
\hline Treatments & $\begin{array}{c}1^{\text {st }} \\
\text { month }\end{array}$ & $\begin{array}{c}2^{\text {nd }} \\
\text { month }\end{array}$ & $\begin{array}{c}3^{\text {rd }} \\
\text { month }\end{array}$ & $\begin{array}{c}4^{\text {th }} \\
\text { month }\end{array}$ & $\begin{array}{c}5^{\text {th }} \\
\text { month }\end{array}$ & $\begin{array}{c}6^{\text {th }} \\
\text { month }\end{array}$ \\
\hline \multicolumn{7}{|c|}{ Storage conditions (S) } \\
\hline Ambient $\left(\mathbf{S}_{1}\right)$ & 4.90 & 4.73 & 0.00 & 0.00 & 0.00 & 0.00 \\
\hline Refrigerated $\left(\mathbf{S}_{2}\right)$ & 6.54 & 5.71 & 5.32 & 4.99 & 4.71 & 4.60 \\
\hline S.E. \pm & 0.11 & 0.10 & 0.03 & 0.04 & 0.05 & 0.04 \\
\hline C.D.@ 5\% & 0.32 & 0.29 & 0.10 & 0.11 & 0.15 & 0.12 \\
\hline \multicolumn{7}{|c|}{ Storage Containers (C) } \\
\hline Tin box $\left(C_{1}\right)$ & 5.42 & 4.90 & 2.58 & 2.35 & 2.20 & 2.15 \\
\hline Glass bottle $\left(C_{2}\right)$ & 5.60 & 5.07 & 2.60 & 2.45 & 2.30 & 2.25 \\
\hline $\begin{array}{c}\text { Polyethylene bag } 200 \text { gauge } \\
\left(C_{3}\right)\end{array}$ & 5.80 & 5.10 & 2.65 & 2.50 & 2.35 & 2.30 \\
\hline $\begin{array}{c}\text { Polyethylene bag } 400 \text { gauge } \\
\left(\mathrm{C}_{4}\right)\end{array}$ & 5.95 & 5.25 & 2.70 & 2.55 & 2.40 & 2.35 \\
\hline $\begin{array}{c}\text { Vaccum packed polyethylene } \\
\text { bag }\left(\mathbf{C}_{5}\right)\end{array}$ & 6.03 & 5.60 & 2.75 & 2.60 & 2.50 & 2.45 \\
\hline Muslin cloth $\left(\mathbf{C}_{6}\right)$ & 5.13 & 4.80 & 2.50 & 2.30 & 2.10 & 2.05 \\
\hline Aluminium foil $\left(\mathbf{C}_{7}\right)$ & 6.10 & 5.83 & 2.85 & 2.70 & 2.65 & 2.55 \\
\hline S.E. \pm & 0.08 & 0.08 & 0.03 & 0.030 & 0.04 & 0.03 \\
\hline C.D.@ 5\% & 0.24 & 0.22 & 0.08 & 0.086 & 0.11 & 0.09 \\
\hline \multicolumn{7}{|c|}{ Interaction effect(SxC) } \\
\hline $\mathrm{S}_{1} \mathrm{C}_{1}$ & 4.63 & 4.40 & 0.00 & 0.00 & 0.00 & 0.00 \\
\hline $\mathrm{S}_{1} \mathrm{C}_{2}$ & 4.80 & 4.67 & 0.00 & 0.00 & 0.00 & 0.00 \\
\hline $\mathrm{S}_{1} \mathrm{C}_{3}$ & 5.00 & 4.70 & 0.00 & 0.00 & 0.00 & 0.00 \\
\hline $\mathbf{S}_{1} \mathbf{C}_{4}$ & 5.10 & 4.80 & 0.00 & 0.00 & 0.00 & 0.00 \\
\hline $\mathrm{S}_{1} \mathrm{C}_{5}$ & 5.17 & 5.10 & 0.00 & 0.00 & 0.00 & 0.00 \\
\hline$S_{1} C_{6}$ & 4.40 & 4.30 & 0.00 & 0.00 & 0.00 & 0.00 \\
\hline $\mathbf{S}_{1} \mathbf{C}_{7}$ & 5.20 & 5.17 & 0.00 & 0.00 & 0.00 & 0.00 \\
\hline $\mathbf{S}_{2} \mathbf{C}_{1}$ & 6.20 & 5.40 & 5.17 & 4.70 & 4.40 & 4.30 \\
\hline $\mathrm{S}_{2} \mathrm{C}_{2}$ & 6.40 & 5.47 & 5.20 & 4.90 & 4.60 & 4.50 \\
\hline $\mathbf{S}_{2} \mathbf{C}_{3}$ & 6.60 & 5.50 & 5.30 & 5.00 & 4.70 & 4.60 \\
\hline $\mathbf{S}_{2} \mathbf{C}_{4}$ & 6.80 & 5.70 & 5.40 & 5.10 & 4.80 & 4.70 \\
\hline $\mathbf{S}_{2} \mathbf{C}_{5}$ & 6.90 & 6.10 & 5.50 & 5.20 & 5.00 & 4.90 \\
\hline$S_{2} C_{6}$ & 5.87 & 5.30 & 5.00 & 4.60 & 4.20 & 4.10 \\
\hline $\mathbf{S}_{2} \mathbf{C}_{7}$ & 7.00 & 6.50 & 5.70 & 5.40 & 5.30 & 5.10 \\
\hline S.E. \pm & 0.29 & 0.27 & 0.09 & 0.10 & 0.13 & 0.11 \\
\hline C.D. @ 5\% & NS & NS & 0.27 & 0.30 & 0.39 & 0.32 \\
\hline
\end{tabular}


Table.5 Effect of storage conditions and containers on root length of seedlings at transplanting (cm) after six months of stored China aster seeds.

\begin{tabular}{|c|c|c|c|c|c|c|}
\hline Treatments & $\begin{array}{c}1^{\text {st }} \\
\text { month }\end{array}$ & $\begin{array}{c}2^{\text {nd }} \\
\text { month }\end{array}$ & $\begin{array}{c}3^{\text {rd }} \\
\text { month }\end{array}$ & $\begin{array}{c}4^{\text {th }} \\
\text { month }\end{array}$ & $\begin{array}{c}5^{\text {th }} \\
\text { month }\end{array}$ & $\begin{array}{c}6^{\text {th }} \\
\text { month }\end{array}$ \\
\hline \multicolumn{7}{|c|}{ Storage conditions (S) } \\
\hline Ambient $\left(\mathbf{S}_{1}\right)$ & 2.62 & 2.29 & 0.00 & 0.00 & 0.00 & 0.00 \\
\hline Refrigerated $\left(\mathbf{S}_{2}\right)$ & 4.15 & 3.66 & 3.56 & 3.41 & 3.30 & 3.21 \\
\hline S.E. \pm & 0.07 & 0.06 & 0.03 & 0.03 & 0.03 & 0.03 \\
\hline C.D.@ 5\% & 0.21 & 0.19 & 0.10 & 0.08 & 0.09 & 0.10 \\
\hline \multicolumn{7}{|c|}{ Storage Containers (C) } \\
\hline Tin box $\left(C_{1}\right)$ & 3.10 & 2.63 & 1.68 & 1.63 & 1.58 & 1.50 \\
\hline Glass bottle $\left(C_{2}\right)$ & 3.37 & 2.87 & 1.72 & 1.65 & 1.60 & 1.58 \\
\hline $\begin{array}{c}\text { Polyethylene bag } 200 \text { gauge } \\
\left(C_{3}\right)\end{array}$ & 3.42 & 3.02 & 1.78 & 1.72 & 1.63 & 1.60 \\
\hline $\begin{array}{c}\text { Polyethylene bag } 400 \text { gauge } \\
\left(\mathrm{C}_{4}\right)\end{array}$ & 3.52 & 3.13 & 1.80 & 1.75 & 1.70 & 1.65 \\
\hline $\begin{array}{c}\text { Vaccum packed polyethylene } \\
\text { bag }\left(\mathbf{C}_{5}\right)\end{array}$ & 3.62 & 3.28 & 1.90 & 1.80 & 1.75 & 1.72 \\
\hline Muslin cloth $\left(\mathrm{C}_{6}\right)$ & 2.97 & 2.50 & 1.60 & 1.55 & 1.50 & 1.45 \\
\hline Aluminium foil $\left(\mathbf{C}_{7}\right)$ & 3.73 & 3.40 & 1.97 & 1.85 & 1.80 & 1.75 \\
\hline S.E. \pm & 0.06 & 0.05 & 0.03 & 0.02 & 0.02 & 0.03 \\
\hline C.D.@5\% & 0.16 & 0.14 & 0.08 & 0.06 & 0.07 & 0.08 \\
\hline \multicolumn{7}{|c|}{ Interaction effect(SxC) } \\
\hline$S_{1} C_{1}$ & 2.43 & 1.77 & 0.00 & 0.00 & 0.00 & 0.00 \\
\hline$S_{1} C_{2}$ & 2.50 & 2.13 & 0.00 & 0.00 & 0.00 & 0.00 \\
\hline $\mathrm{S}_{1} \mathrm{C}_{3}$ & 2.57 & 2.40 & 0.00 & 0.00 & 0.00 & 0.00 \\
\hline $\mathrm{S}_{1} \mathrm{C}_{4}$ & 2.70 & 2.50 & 0.00 & 0.00 & 0.00 & 0.00 \\
\hline $\mathrm{S}_{1} \mathrm{C}_{5}$ & 2.83 & 2.73 & 0.00 & 0.00 & 0.00 & 0.00 \\
\hline$S_{1} C_{6}$ & 2.40 & 1.70 & 0.00 & 0.00 & 0.00 & 0.00 \\
\hline $\mathrm{S}_{1} \mathbf{C}_{7}$ & 2.93 & 2.80 & 0.00 & 0.00 & 0.00 & 0.00 \\
\hline $\mathbf{S}_{2} \mathbf{C}_{1}$ & 3.77 & 3.50 & 3.37 & 3.27 & 3.17 & 3.00 \\
\hline $\mathbf{S}_{2} \mathbf{C}_{2}$ & 4.23 & 3.60 & 3.43 & 3.30 & 3.20 & 3.17 \\
\hline $\mathrm{S}_{2} \mathrm{C}_{3}$ & 4.27 & 3.63 & 3.57 & 3.43 & 3.27 & 3.20 \\
\hline $\mathrm{S}_{2} \mathrm{C}_{4}$ & 4.33 & 3.77 & 3.60 & 3.50 & 3.40 & 3.30 \\
\hline $\mathrm{S}_{2} \mathrm{C}_{5}$ & 4.40 & 3.83 & 3.80 & 3.60 & 3.50 & 3.43 \\
\hline$S_{2} C_{6}$ & 3.53 & 3.30 & 3.20 & 3.10 & 3.00 & 2.90 \\
\hline $\mathbf{S}_{2} \mathbf{C}_{7}$ & 4.53 & 4.00 & 3.93 & 3.70 & 3.60 & 3.50 \\
\hline S.E. \pm & 0.19 & 0.17 & 0.09 & 0.07 & 0.08 & 0.09 \\
\hline C.D. @ 5\% & NS & NS & 0.26 & 0.21 & 0.24 & 0.27 \\
\hline
\end{tabular}


Table.6 Effect of storage conditions and containers on shoot weight $(\mathrm{g})$ after six months of stored China aster seeds.

\begin{tabular}{|c|c|c|c|c|c|c|}
\hline Treatments & $\begin{array}{c}1^{\text {st }} \\
\text { month }\end{array}$ & $\begin{array}{c}2^{\text {nd }} \\
\text { month }\end{array}$ & $\begin{array}{c}3^{\text {rd }} \\
\text { month }\end{array}$ & $\begin{array}{c}4^{\text {th }} \\
\text { month }\end{array}$ & $\begin{array}{c}5^{\text {th }} \\
\text { month }\end{array}$ & $\begin{array}{c}6^{\text {th }} \\
\text { month }\end{array}$ \\
\hline \multicolumn{7}{|c|}{ Storage conditions (S) } \\
\hline Ambient $\left(\mathbf{S}_{1}\right)$ & 0.36 & 0.34 & 0.00 & 0.00 & 0.00 & 0.00 \\
\hline Refrigerated $\left(\mathbf{S}_{2}\right)$ & 0.38 & 0.36 & 0.33 & 0.32 & 0.31 & 0.28 \\
\hline S.E. \pm & 0.005 & 0.007 & 0.006 & 0.005 & 0.005 & 0.004 \\
\hline C.D.@ 5\% & 0.014 & 0.020 & 0.017 & 0.015 & 0.014 & 0.013 \\
\hline \multicolumn{7}{|c|}{ Storage Containers (C) } \\
\hline Tin box $\left(C_{1}\right)$ & 0.36 & 0.32 & 0.15 & 0.14 & 0.14 & 0.13 \\
\hline Glass bottle $\left(C_{2}\right)$ & 0.36 & 0.34 & 0.16 & 0.16 & 0.15 & 0.14 \\
\hline $\begin{array}{c}\text { Polyethylene bag } 200 \text { gauge } \\
\left(C_{3}\right)\end{array}$ & 0.37 & 0.35 & 0.16 & 0.16 & 0.15 & 0.14 \\
\hline $\begin{array}{c}\text { Polyethylene bag } 400 \text { gauge } \\
\left(\mathrm{C}_{4}\right)\end{array}$ & 0.38 & 0.36 & 0.17 & 0.17 & 0.16 & 0.15 \\
\hline $\begin{array}{c}\text { Vaccum packed polyethylene } \\
\text { bag }\left(\mathbf{C}_{5}\right)\end{array}$ & 0.39 & 0.37 & 0.18 & 0.18 & 0.17 & 0.15 \\
\hline Muslin cloth $\left(\mathrm{C}_{6}\right)$ & 0.34 & 0.30 & 0.14 & 0.13 & 0.13 & 0.12 \\
\hline Aluminium foil $\left(\mathbf{C}_{7}\right)$ & 0.40 & 0.38 & 0.19 & 0.18 & 0.17 & 0.16 \\
\hline S.E. \pm & 0.004 & 0.005 & 0.004 & 0.004 & 0.004 & 0.003 \\
\hline C.D. @ 5\% & 0.011 & 0.015 & 0.013 & 0.012 & 0.010 & 0.010 \\
\hline \multicolumn{7}{|c|}{ Interaction effect(SxC) } \\
\hline$S_{1} C_{1}$ & 0.35 & 0.32 & 0.00 & 0.00 & 0.00 & 0.00 \\
\hline $\mathrm{S}_{1} \mathrm{C}_{2}$ & 0.36 & 0.33 & 0.00 & 0.00 & 0.00 & 0.00 \\
\hline $\mathrm{S}_{1} \mathrm{C}_{3}$ & 0.36 & 0.34 & 0.00 & 0.00 & 0.00 & 0.00 \\
\hline $\mathrm{S}_{1} \mathrm{C}_{4}$ & 0.36 & 0.35 & 0.00 & 0.00 & 0.00 & 0.00 \\
\hline $\mathrm{S}_{1} \mathrm{C}_{5}$ & 0.37 & 0.35 & 0.00 & 0.00 & 0.00 & 0.00 \\
\hline$S_{1} C_{6}$ & 0.33 & 0.30 & 0.00 & 0.00 & 0.00 & 0.00 \\
\hline $\mathbf{S}_{1} \mathbf{C}_{7}$ & 0.39 & 0.36 & 0.00 & 0.00 & 0.00 & 0.00 \\
\hline $\mathbf{S}_{2} \mathbf{C}_{1}$ & 0.36 & 0.32 & 0.29 & 0.29 & 0.28 & 0.27 \\
\hline $\mathbf{S}_{2} \mathbf{C}_{2}$ & 0.37 & 0.34 & 0.32 & 0.31 & 0.30 & 0.27 \\
\hline $\mathrm{S}_{2} \mathrm{C}_{3}$ & 0.38 & 0.37 & 0.33 & 0.32 & 0.31 & 0.29 \\
\hline $\mathbf{S}_{2} \mathbf{C}_{4}$ & 0.39 & 0.38 & 0.34 & 0.33 & 0.32 & 0.30 \\
\hline $\mathrm{S}_{2} \mathrm{C}_{5}$ & 0.42 & 0.39 & 0.36 & 0.35 & 0.34 & 0.30 \\
\hline$S_{2} C_{6}$ & 0.35 & 0.30 & 0.28 & 0.27 & 0.25 & 0.24 \\
\hline $\mathbf{S}_{2} \mathbf{C}_{7}$ & 0.42 & 0.41 & 0.39 & 0.37 & 0.35 & 0.33 \\
\hline S.E. \pm & 0.013 & 0.018 & 0.016 & 0.014 & 0.012 & 0.011 \\
\hline C.D. @ 5\% & NS & NS & 0.045 & 0.040 & 0.036 & 0.033 \\
\hline
\end{tabular}

NS-Non Significant 
Table.7 Effect of storage conditions and containers on root weight (g) after six months of stored China aster seeds.

\begin{tabular}{|c|c|c|c|c|c|c|}
\hline Treatments & $\begin{array}{c}1^{\text {st }} \\
\text { month }\end{array}$ & $\begin{array}{c}2^{\text {nd }} \\
\text { month }\end{array}$ & $\begin{array}{c}3^{\text {rd }} \\
\text { month }\end{array}$ & $\begin{array}{c}4^{\text {th }} \\
\text { month }\end{array}$ & $\begin{array}{c}5^{\text {th }} \\
\text { month }\end{array}$ & $\begin{array}{c}6^{\text {th }} \\
\text { month }\end{array}$ \\
\hline \multicolumn{7}{|c|}{ Storage conditions (S) } \\
\hline Ambient $\left(\mathbf{S}_{1}\right)$ & 0.030 & 0.027 & 0.000 & 0.000 & 0.000 & 0.000 \\
\hline Refrigerated $\left(\mathbf{S}_{2}\right)$ & 0.034 & 0.030 & 0.026 & 0.025 & 0.023 & 0.021 \\
\hline S.E. \pm & 0.0007 & 0.0009 & 0.0004 & 0.00062 & 0.0005 & 0.0005 \\
\hline C.D.@ 5\% & 0.0019 & 0.0026 & 0.0013 & 0.00181 & 0.0016 & 0.0015 \\
\hline \multicolumn{7}{|c|}{ Storage Containers (C) } \\
\hline Tin box $\left(C_{1}\right)$ & 0.029 & 0.024 & 0.010 & 0.011 & 0.010 & 0.009 \\
\hline Glass bottle $\left(C_{2}\right)$ & 0.030 & 0.026 & 0.013 & 0.012 & 0.011 & 0.010 \\
\hline Polyethylene bag 200 gauge $\left(C_{3}\right)$ & 0.032 & 0.029 & 0.013 & 0.013 & 0.012 & 0.011 \\
\hline Polyethylene bag 400 gauge $\left(C_{4}\right)$ & 0.032 & 0.031 & 0.014 & 0.014 & 0.013 & 0.011 \\
\hline Vaccum packed polyethylene bag $\left(C_{5}\right)$ & 0.036 & 0.033 & 0.015 & 0.015 & 0.014 & 0.012 \\
\hline Muslin cloth $\left(C_{6}\right)$ & 0.027 & 0.021 & 0.010 & 0.009 & 0.009 & 0.008 \\
\hline Aluminium foil $\left(\mathbf{C}_{7}\right)$ & 0.037 & 0.034 & 0.017 & 0.016 & 0.014 & 0.013 \\
\hline S.E. \pm & 0.0005 & 0.0007 & 0.0003 & 0.00048 & 0.0004 & 0.0004 \\
\hline C.D.@ 5\% & 0.0015 & 0.0020 & 0.0010 & 0.00138 & 0.0012 & 0.0011 \\
\hline \multicolumn{7}{|c|}{ Interaction effect(SxC) } \\
\hline$S_{1} C_{1}$ & 0.028 & 0.024 & 0.000 & 0.000 & 0.000 & 0.000 \\
\hline$S_{1} C_{2}$ & 0.029 & 0.026 & 0.000 & 0.000 & 0.000 & 0.000 \\
\hline $\mathrm{S}_{1} \mathrm{C}_{3}$ & 0.030 & 0.027 & 0.000 & 0.000 & 0.000 & 0.000 \\
\hline$S_{1} C_{4}$ & 0.031 & 0.029 & 0.000 & 0.000 & 0.000 & 0.000 \\
\hline $\mathrm{S}_{1} \mathrm{C}_{5}$ & 0.032 & 0.030 & 0.000 & 0.000 & 0.000 & 0.000 \\
\hline$S_{1} C_{6}$ & 0.025 & 0.021 & 0.000 & 0.000 & 0.000 & 0.000 \\
\hline $\mathrm{S}_{1} \mathrm{C}_{7}$ & 0.034 & 0.031 & 0.000 & 0.000 & 0.000 & 0.000 \\
\hline $\mathrm{S}_{2} \mathrm{C}_{1}$ & 0.030 & 0.024 & 0.021 & 0.021 & 0.019 & 0.018 \\
\hline $\mathbf{S}_{2} \mathbf{C}_{2}$ & 0.031 & 0.027 & 0.025 & 0.023 & 0.022 & 0.019 \\
\hline $\mathbf{S}_{2} \mathbf{C}_{3}$ & 0.033 & 0.031 & 0.026 & 0.025 & 0.024 & 0.022 \\
\hline $\mathrm{S}_{2} \mathrm{C}_{4}$ & 0.034 & 0.032 & 0.028 & 0.027 & 0.025 & 0.023 \\
\hline $\mathrm{S}_{2} \mathrm{C}_{5}$ & 0.039 & 0.035 & 0.030 & 0.029 & 0.027 & 0.024 \\
\hline $\mathrm{S}_{2} \mathrm{C}_{6}$ & 0.028 & 0.022 & 0.019 & 0.018 & 0.017 & 0.016 \\
\hline $\mathbf{S}_{2} \mathbf{C}_{7}$ & 0.040 & 0.038 & 0.033 & 0.031 & 0.029 & 0.027 \\
\hline S.E. \pm & 0.0017 & 0.0024 & 0.0012 & 0.00165 & 0.0014 & 0.0014 \\
\hline C.D.@ $@$ 5\% & NS & NS & 0.0034 & 0.00479 & 0.0042 & 0.0039 \\
\hline
\end{tabular}


Table.8 Effect of storage conditions and containers on root:shoot ratio after six months of stored China aster seeds.

\begin{tabular}{|c|c|c|c|c|c|c|}
\hline Treatments & $\begin{array}{c}1^{\text {st }} \\
\text { month }\end{array}$ & $\begin{array}{c}2^{\text {nd }} \\
\text { month }\end{array}$ & $\begin{array}{c}3^{\text {rd }} \\
\text { month }\end{array}$ & $\begin{array}{c}4^{\text {th }} \\
\text { month }\end{array}$ & $\begin{array}{c}5^{\text {th }} \\
\text { month }\end{array}$ & $\begin{array}{c}6^{\text {th }} \\
\text { month }\end{array}$ \\
\hline \multicolumn{7}{|c|}{ Storage conditions (S) } \\
\hline Ambient $\left(S_{1}\right)$ & 0.08 & 0.08 & 0.00 & 0.00 & 0.00 & 0.00 \\
\hline Refrigerated $\left(\mathbf{S}_{2}\right)$ & 0.09 & 0.08 & 0.08 & 0.08 & 0.08 & 0.07 \\
\hline S.E. \pm & 0.0010 & 0.0008 & 0.0006 & 0.0007 & 0.0008 & 0.0005 \\
\hline C.D.@ 5\% & 0.0030 & 0.0024 & 0.0017 & 0.0019 & 0.0024 & 0.0016 \\
\hline \multicolumn{7}{|c|}{ Storage Containers (C) } \\
\hline Tin box $\left(C_{1}\right)$ & 0.082 & 0.074 & 0.036 & 0.035 & 0.035 & 0.034 \\
\hline Glass bottle $\left(C_{2}\right)$ & 0.084 & 0.079 & 0.039 & 0.037 & 0.037 & 0.034 \\
\hline $\begin{array}{c}\text { Polyethylene bag } 200 \text { gauge } \\
\left(C_{3}\right)\end{array}$ & 0.085 & 0.082 & 0.040 & 0.039 & 0.039 & 0.038 \\
\hline $\begin{array}{c}\text { Polyethylene bag } 400 \text { gauge } \\
\left(\mathrm{C}_{4}\right)\end{array}$ & 0.086 & 0.084 & 0.042 & 0.040 & 0.040 & 0.038 \\
\hline $\begin{array}{c}\text { Vaccum packed polyethylene } \\
\text { bag }\left(\mathbf{C}_{5}\right)\end{array}$ & 0.090 & 0.087 & 0.042 & 0.041 & 0.040 & 0.038 \\
\hline Muslin cloth $\left(\mathrm{C}_{6}\right)$ & 0.079 & 0.071 & 0.035 & 0.034 & 0.034 & 0.034 \\
\hline Aluminium foil $\left(\mathrm{C}_{7}\right)$ & 0.092 & 0.089 & 0.043 & 0.042 & 0.041 & 0.041 \\
\hline S.E. \pm & 0.0008 & 0.0006 & 0.0005 & 0.0005 & 0.0006 & 0.0004 \\
\hline C.D.@5\% & 0.0023 & 0.0018 & 0.0013 & 0.0015 & 0.0018 & 0.0012 \\
\hline \multicolumn{7}{|c|}{ Interaction effect(SxC) } \\
\hline$S_{1} C_{1}$ & 0.081 & 0.074 & 0.000 & 0.000 & 0.000 & 0.000 \\
\hline$S_{1} C_{2}$ & 0.083 & 0.078 & 0.000 & 0.000 & 0.000 & 0.000 \\
\hline $\mathrm{S}_{1} \mathrm{C}_{3}$ & 0.084 & 0.080 & 0.000 & 0.000 & 0.000 & 0.000 \\
\hline $\mathrm{S}_{1} \mathrm{C}_{4}$ & 0.084 & 0.084 & 0.000 & 0.000 & 0.000 & 0.000 \\
\hline $\mathrm{S}_{1} \mathrm{C}_{5}$ & 0.086 & 0.085 & 0.000 & 0.000 & 0.000 & 0.000 \\
\hline$S_{1} C_{6}$ & 0.076 & 0.070 & 0.000 & 0.000 & 0.000 & 0.000 \\
\hline $\mathbf{S}_{1} \mathbf{C}_{7}$ & 0.089 & 0.086 & 0.000 & 0.000 & 0.000 & 0.000 \\
\hline $\mathbf{S}_{2} \mathbf{C}_{1}$ & 0.083 & 0.074 & 0.071 & 0.070 & 0.069 & 0.069 \\
\hline $\mathbf{S}_{2} \mathbf{C}_{2}$ & 0.085 & 0.079 & 0.079 & 0.074 & 0.073 & 0.067 \\
\hline $\mathbf{S}_{2} \mathrm{C}_{3}$ & 0.086 & 0.084 & 0.081 & 0.078 & 0.077 & 0.076 \\
\hline $\mathbf{S}_{2} \mathbf{C}_{4}$ & 0.087 & 0.085 & 0.083 & 0.081 & 0.080 & 0.076 \\
\hline $\mathbf{S}_{2} \mathbf{C}_{5}$ & 0.094 & 0.089 & 0.084 & 0.083 & 0.080 & 0.076 \\
\hline $\mathbf{S}_{2} \mathbf{C}_{6}$ & 0.081 & 0.072 & 0.070 & 0.069 & 0.068 & 0.067 \\
\hline $\mathbf{S}_{2} \mathbf{C}_{7}$ & 0.095 & 0.093 & 0.085 & 0.085 & 0.083 & 0.082 \\
\hline S.E. \pm & 0.0027 & 0.0022 & 0.0016 & 0.0018 & 0.0021 & 0.0014 \\
\hline C.D.@ 5\% & NS & NS & 0.0046 & 0.0051 & 0.0062 & 0.0042 \\
\hline
\end{tabular}


Table.9 Effect of storage conditions and containers on germination percentage at laboratory condition after six months of stored China aster seeds.

\begin{tabular}{|c|c|c|c|c|c|c|}
\hline Treatments & $\begin{array}{c}1^{\text {st }} \\
\text { month }\end{array}$ & $\begin{array}{c}2^{\text {nd }} \\
\text { month }\end{array}$ & $\begin{array}{c}3^{\text {rd }} \\
\text { month }\end{array}$ & $\begin{array}{c}4^{\text {th }} \\
\text { month }\end{array}$ & $\begin{array}{c}5^{\text {th }} \\
\text { month }\end{array}$ & $\begin{array}{c}6^{\text {th }} \\
\text { month }\end{array}$ \\
\hline \multicolumn{7}{|c|}{ Storage conditions (S) } \\
\hline Ambient $\left(\mathbf{S}_{1}\right)$ & $\begin{array}{c}38.14 \\
(38.07) \\
*\end{array}$ & $\begin{array}{c}32.33 \\
(34.54)\end{array}$ & $\begin{array}{c}21.95 \\
(27.61)\end{array}$ & $\begin{array}{c}14.19 \\
(21.24)\end{array}$ & $\begin{array}{c}9.57 \\
(16.30)\end{array}$ & $\begin{array}{c}1.57 \\
(2.75)\end{array}$ \\
\hline Refrigerated $\left(\mathbf{S}_{2}\right)$ & $\begin{array}{c}88.05 \\
(70.49)\end{array}$ & $\begin{array}{c}83.67 \\
(66.58)\end{array}$ & $\begin{array}{c}79.71 \\
(63.52)\end{array}$ & $\begin{array}{c}75.24 \\
(60.36)\end{array}$ & $\begin{array}{c}72.81 \\
(58.77)\end{array}$ & $\begin{array}{c}67.71 \\
(55.49)\end{array}$ \\
\hline S.E. \pm & 0.33 & 0.43 & 0.60 & 0.46 & 0.50 & 0.44 \\
\hline C.D.@ 5\% & 0.97 & 1.24 & 1.75 & 1.32 & 1.46 & 1.27 \\
\hline \multicolumn{7}{|c|}{ Storage Containers (C) } \\
\hline Tin box $\left(C_{1}\right)$ & $\begin{array}{c}57.50 \\
(50.07)\end{array}$ & $\begin{array}{c}52.83 \\
(46.92)\end{array}$ & $\begin{array}{c}45.67 \\
(41.97)\end{array}$ & $\begin{array}{c}38.67 \\
(36.50)\end{array}$ & $\begin{array}{c}36.50 \\
(34.32)\end{array}$ & $\begin{array}{c}31.00 \\
(26.00)\end{array}$ \\
\hline Glass bottle $\left(C_{2}\right)$ & $\begin{array}{c}59.50 \\
(51.44)\end{array}$ & $\begin{array}{c}55.00 \\
(48.34)\end{array}$ & $\begin{array}{c}47.17 \\
(43.04)\end{array}$ & $\begin{array}{c}40.83 \\
(38.25)\end{array}$ & $\begin{array}{c}38.83 \\
(36.41)\end{array}$ & $\begin{array}{c}32.50 \\
(26.87)\end{array}$ \\
\hline $\begin{array}{c}\text { Polyethylene bag } 200 \text { gauge } \\
\left(C_{3}\right)\end{array}$ & $\begin{array}{c}60.67 \\
(52.25)\end{array}$ & $\begin{array}{c}56.67 \\
(49.54)\end{array}$ & $\begin{array}{c}50.67 \\
(45.47)\end{array}$ & $\begin{array}{c}44.17 \\
(40.55)\end{array}$ & $\begin{array}{c}42.17 \\
(38.79)\end{array}$ & $\begin{array}{c}34.17 \\
(27.90)\end{array}$ \\
\hline $\begin{array}{c}\text { Polyethylene bag } 400 \text { gauge } \\
\left(\mathrm{C}_{4}\right)\end{array}$ & $\begin{array}{c}64.17 \\
(54.64)\end{array}$ & $\begin{array}{c}58.83 \\
(51.14)\end{array}$ & $\begin{array}{c}52.33 \\
(46.70)\end{array}$ & $\begin{array}{c}47.33 \\
(43.06)\end{array}$ & $\begin{array}{c}43.17 \\
(39.73)\end{array}$ & $\begin{array}{c}35.50 \\
(28.72)\end{array}$ \\
\hline $\begin{array}{l}\text { Vaccum packed polyethylene } \\
\text { bag }\left(\mathbf{C}_{5}\right)\end{array}$ & $\begin{array}{c}70.50 \\
(59.78)\end{array}$ & $\begin{array}{c}65.83 \\
(56.09)\end{array}$ & $\begin{array}{c}55.83 \\
(49.31)\end{array}$ & $\begin{array}{c}50.00 \\
(45.01)\end{array}$ & $\begin{array}{c}45.67 \\
(41.72)\end{array}$ & $\begin{array}{c}36.67 \\
(29.47)\end{array}$ \\
\hline Muslin cloth $\left(\mathrm{C}_{6}\right)$ & $\begin{array}{c}55.67 \\
(48.90)\end{array}$ & $\begin{array}{c}49.33 \\
(44.57)\end{array}$ & $\begin{array}{l}41.50 \\
(38.82)\end{array}$ & $\begin{array}{c}35.00 \\
(32.41)\end{array}$ & $\begin{array}{c}30.50 \\
(25.68)\end{array}$ & $\begin{array}{c}29.17 \\
(24.90)\end{array}$ \\
\hline Aluminium foil $\left(\mathbf{C}_{7}\right)$ & $\begin{array}{c}73.67 \\
(62.87)\end{array}$ & $\begin{array}{c}67.50 \\
(57.31)\end{array}$ & $\begin{array}{c}62.67 \\
(53.65)\end{array}$ & $\begin{array}{c}57.00 \\
(49.85)\end{array}$ & $\begin{array}{c}51.50 \\
(46.10)\end{array}$ & $\begin{array}{c}43.50 \\
(39.98)\end{array}$ \\
\hline S.E. \pm & 0.25 & 0.33 & 0.46 & 0.35 & 0.38 & 0.33 \\
\hline C.D.@ 5\% & 0.74 & 0.95 & 1.33 & 1.01 & 1.11 & 0.97 \\
\hline \multicolumn{7}{|c|}{ Interaction effect (SxC) } \\
\hline $\mathrm{S}_{1} \mathbf{C}_{1}$ & $\begin{array}{c}32.00 \\
(34.44)\end{array}$ & $\begin{array}{c}27.67 \\
(31.72)\end{array}$ & $\begin{array}{c}17.33 \\
(24.58)\end{array}$ & $\begin{array}{c}8.67 \\
(17.00)\end{array}$ & $\begin{array}{c}5.33 \\
(13.27)\end{array}$ & $\begin{array}{c}0.00 \\
(0.00)\end{array}$ \\
\hline $\mathbf{S}_{1} \mathbf{C}_{2}$ & $\begin{array}{c}34.33 \\
(35.87)\end{array}$ & $\begin{array}{c}29.67 \\
(32.98)\end{array}$ & $\begin{array}{c}18.67 \\
(25.56)\end{array}$ & $\begin{array}{c}10.67 \\
(19.01)\end{array}$ & $\begin{array}{c}7.67 \\
(16.02)\end{array}$ & $\begin{array}{c}0.00 \\
(0.00)\end{array}$ \\
\hline $\mathrm{S}_{1} \mathrm{C}_{3}$ & $\begin{array}{c}35.33 \\
(36.47)\end{array}$ & $\begin{array}{c}30.67 \\
(33.62)\end{array}$ & $\begin{array}{c}21.00 \\
(27.24)\end{array}$ & $\begin{array}{c}12.00 \\
(20.19)\end{array}$ & $\begin{array}{c}8.67 \\
(17.08)\end{array}$ & $\begin{array}{c}0.00 \\
(0.00)\end{array}$ \\
\hline $\mathbf{S}_{1} \mathbf{C}_{4}$ & $\begin{array}{c}40.00 \\
(39.23)\end{array}$ & $\begin{array}{c}31.67 \\
(34.24)\end{array}$ & $\begin{array}{c}22.00 \\
(27.97)\end{array}$ & $\begin{array}{c}16.67 \\
(24.07)\end{array}$ & $\begin{array}{c}10.33 \\
(18.72)\end{array}$ & $\begin{array}{c}0.00 \\
(0.00)\end{array}$ \\
\hline $\mathrm{S}_{1} \mathrm{C}_{5}$ & $\begin{array}{c}46.33 \\
(42.90)\end{array}$ & $\begin{array}{c}40.67 \\
(39.62)\end{array}$ & $\begin{array}{c}25.33 \\
(30.21)\end{array}$ & $\begin{array}{c}18.67 \\
(25.58)\end{array}$ & $\begin{array}{c}12.67 \\
(20.83)\end{array}$ & $\begin{array}{c}0.00 \\
(0.00)\end{array}$ \\
\hline$S_{1} C_{6}$ & $\begin{array}{c}29.00 \\
(32.58)\end{array}$ & $\begin{array}{c}23.00 \\
(28.63)\end{array}$ & $\begin{array}{c}12.00 \\
(20.19)\end{array}$ & $\begin{array}{c}3.00 \\
(9.88)\end{array}$ & $\begin{array}{c}0.00 \\
(0.00)\end{array}$ & $\begin{array}{c}0.00 \\
(0.00)\end{array}$ \\
\hline $\mathrm{S}_{1} \mathbf{C}_{7}$ & $\begin{array}{c}50.00 \\
(45.00)\end{array}$ & $\begin{array}{c}43.00 \\
(40.97)\end{array}$ & $\begin{array}{c}37.33 \\
(37.52)\end{array}$ & $\begin{array}{c}29.67 \\
(32.98)\end{array}$ & $\begin{array}{c}22.33 \\
(28.16)\end{array}$ & $\begin{array}{c}11.00 \\
(19.28)\end{array}$ \\
\hline
\end{tabular}




\begin{tabular}{|c|c|c|c|c|c|c|}
\hline $\mathbf{S}_{\mathbf{2}} \mathbf{C}_{\mathbf{1}}$ & 83.00 & 78.00 & 74.00 & 68.67 & 67.67 & 62.00 \\
& $(65.70)$ & $(62.11)$ & $(59.35)$ & $(55.99)$ & $(55.37)$ & $(52.01)$ \\
\hline $\mathbf{S}_{\mathbf{2}} \mathbf{C}_{\mathbf{2}}$ & 84.67 & 80.33 & 75.67 & 71.00 & 70.00 & 65.00 \\
& $(67.02)$ & $(63.69)$ & $(60.51)$ & $(57.48)$ & $(56.80)$ & $(53.74)$ \\
\hline $\mathbf{S}_{\mathbf{2}} \mathbf{C}_{\mathbf{3}}$ & 86.00 & 82.67 & 80.33 & 76.33 & 75.67 & 68.33 \\
& $(68.04)$ & $(65.47)$ & $(63.70)$ & $(60.91)$ & $(60.51)$ & $(55.80)$ \\
\hline $\mathbf{S}_{\mathbf{2}} \mathbf{C}_{\mathbf{4}}$ & 88.33 & 86.00 & 82.67 & 78.00 & 76.00 & 71.00 \\
& $(70.05)$ & $(68.04)$ & $(65.43)$ & $(62.04)$ & $(60.74)$ & $(57.45)$ \\
\hline $\mathbf{S}_{\mathbf{2}} \mathbf{C}_{\mathbf{5}}$ & 94.67 & 91.00 & 86.33 & 81.33 & 78.67 & 73.33 \\
& $(76.66)$ & $(72.56)$ & $(68.40)$ & $(64.45)$ & $(62.61)$ & $(58.93)$ \\
\hline $\mathbf{S}_{\mathbf{2}} \mathbf{C}_{\mathbf{6}}$ & 82.33 & 75.67 & 71.00 & 67.00 & 61.00 & 58.33 \\
& $(65.22)$ & $(60.51)$ & $(57.45)$ & $(54.95)$ & $(51.36)$ & $(49.80)$ \\
\hline $\mathbf{S}_{\mathbf{2}} \mathbf{C}_{\mathbf{7}}$ & 97.33 & 92.00 & 88.00 & 84.33 & 80.67 & 76.00 \\
& $(80.73)$ & $(73.65)$ & $(69.77)$ & $(66.72)$ & $(64.04)$ & $(60.68)$ \\
\hline $\mathbf{S . E . \pm}$ & 0.88 & 1.13 & 1.59 & 1.21 & 1.33 & 1.16 \\
\hline C.D. @ 5\% & $\mathrm{NS}$ & $\mathrm{NS}$ & $\mathrm{NS}$ & 3.50 & 3.85 & 3.36 \\
\hline
\end{tabular}

\section{Interaction effect $(\mathrm{SxC})$}

China aster seeds stored for six months showed non-significant differences for all the observations except percent seed germination in laboratory for first two months and thereafter significant differences were observed from third month to sixth month due to interaction effect of storage conditions and containers of six months stored seeds of China aster. Non-significant differences in percent seed germination in laboratory were observed for first three months and thereafter significant differences were observed from fourth to sixth months.

Numerically seeds stored in aluminium foil under refrigerated condition $\left(\mathrm{S}_{2} \mathrm{C}_{7}\right)$ recorded higher seed quality parameters viz. per cent seed germination at field level (92.67 and $72.00 \%)$, seedling survival percentage (89.33 and $70.67 \%$ ), height of seedling (11.53 and $8.60 \mathrm{~cm}$ ), shoot length $(7.0$ and $5.10 \mathrm{~cm})$, root length (4.53 and $3.50 \mathrm{~cm})$, shoot weight $(0.42$ and $0.33 \mathrm{~g})$, root weight $(0.040$ and $0.027 \mathrm{~g})$, root: shoot ratio (0.095 and 0.082) and percent seed germination at laboratory level (97.33 and $76.00 \%$ ) followed by vacuum packed polyethylene bag stored under refrigerated condition $\left(\mathrm{S}_{2} \mathrm{C}_{5}\right)$ which showed per cent seed germination at field level (89.33 and 69.67\%), seedling survival percentage (87.33 and 67.33 $\%)$, height of seedling $(11.30$ and $8.33 \mathrm{~cm})$, shoot length $(6.90$ and $4.90 \mathrm{~cm})$, root length $(4.40$ and $3.43 \mathrm{~cm})$, shoot weight $(0.42$ and $0.30 \mathrm{~g})$, root weight $(0.039$ and $0.024 \mathrm{~g}$ ), root: shoot ratio (0.094 and 0.076) and percent seed germination at laboratory level (94.67 and $73.33 \%$ ) for first and sixth month, respectively (Table 1, 2, 3, 4, 5, 6, 7, 8 and 9). While it was lowest in seed stored in cloth bag and kept under ambient conditions. For all the observations seeds stored in aluminium foil kept at refrigerated condition $\left(\mathrm{S}_{2} \mathrm{C}_{7}\right)$ were statistically at par with vacuum packed polyethylene bag kept at refrigerated condition $\left(\mathrm{S}_{2} \mathrm{C}_{5}\right)$ for third, fourth, fifth and sixth month.

However, decrease in all these parameters with increase in storage period has been noticed. These results are in conformity with Singh et al., (2004) in marigold, Tang et al., (2009) in Liguralia virgaurea. Whereas with course of time decline in seed quality parameters could be due to increase in fat acidity, ultra structural changes, reduced activity of enzymes and weakening of 
membrane integrity. This also might be due to the ageing phenomenon and due to the depletion of food reserves and decline in synthetic activity (Shakuntala et al., 2012). Agrawal (1990) reported that in orthodox seeds, decrease in the activity of enzymes during storage may be due to decrease in the activity of PEP and RUBP carboxylase and an increase in protease activity. The weakening of membrane integrity lead to increased leaching of water soluble sugars and leucine ${ }^{14} \mathrm{C}$ with seed deterioration. The reduction in viability during longer storage period is also due to the evolution of volatile compounds such as acetaldehyde, methanol, ethanol and acetone. (Zhang et al., 1994).

However, seeds stored in aluminium foil kept at refrigerated $\mathrm{S}_{2} \mathrm{C}_{7}$ condition exhibited relatively higher percent seed germination as well as seedling growth parameters compared to other interactions. These results are in line with the findings of Yogeesha et al., in China aster, Dhatt and Kumar (2010c) in Coreopsis lanceolata where laminated aluminium foil packaging at cold storage resulted in higher per cent seed germination and seedling parameters.

The seeds of China aster stored in aluminium foil kept under refrigerated storage conditions retained highest germination and seedling parameters after one year of storage period and this treatment can be used for storage in order to maintain viability of China aster seeds for one year.

\section{References}

Agrawal, P. K. 1990. Seed deterioration during storage. Proceedings of the International Congress of Plant Physiology, New Delhi, India.2:12711278.

Anonymous. 2006. International Rules for Seed Testing. Seed Science and
Technology, 24 (Suppl.):23-46.

Bharathi, D. 2002. Influence of seed treatments and packaging materials on storability of Gaillardia cv. DGS-1 M.Sc. (Agri.) Thesis, University of Agricultural Sciences, Dharwad.

Cicek, E., Cetin, B., Ozbayram, A. K. and Turkylmaz, H. 1994. Effect of dessication, temperatureand storage on germination of snow flake (Leucojum aestivum L.) seeds. ArtvinCoruh University Journal of Forestry, 14(2):245-252.

Deepa, S. Paramesh, R., Reddy, D. V., Jayanthi, R., and Doss, D. D., 2008. Influence of packaging materials on storability of China aster cv. Poornima under ambient condition. Environment and Ecology, 26(3A):1253-1255.

Dhatt, K. K. and Kumar, R. 2009. Effect of storage conditions, packaging material and storage period on seed germination and seed viability of Gazania splendens L. Seed Research, 37(1/2):88-98

Dhatt, K. K., and Kumar, R. 2010a. Effect of storage temperature and packaging material on seed germination and seed viability of Gaillardia. Indian Journal of Horticulture, 67(3):362-371.

Dhatt, K. K., and Ramesh Kumar 2010b. Effect of storage temperature and packaging material on seed germination of Matthiola (Matthiola incana). Environment and Ecology, 28(4A):2632-2636.

Dhatt, K. K., and Ramesh Kumar 2010c. Effect of storage conditions, packaging and storage period on seed germination and viability of Coreopsis lanceolata L. Advances in Horticultural Science, 24(3):163-168.

Dubey, R. K., Ramesh Kumar, and Poonam. 2002. Effect of storage materials on seed germination and seed viability in Cosmos. Journal of Ornamental 
Horticulture, 5(2):51-52.

Floris, C.1970. Ageing in Triticum durum seeds: behavior of embryos and endosperms from aged seeds as revealed by the embryo transplantation technique. Journal of Experimental Botany, 21:462-468.

Gnyandev, B. 2006. Effect of pinching, plant nutrition and growth retardant sprays on seed yield, quality and storage studies in China aster [Callistephus chinensis (L.) Nees]. M.Sc. (Agri.) Thesis, University of Agricultural Sciences, Dharwad.

Gujar, A. R., Nimbalkar, C. A. and Dumbre, S. S. 2001. Studies on viability of China aster seeds. Journal of Maharashtra Agricultural Universities, 26 (1):77-79.

Harrington, J. F. 1972. Biochemical basis of seed longevity. Seed Science and Technology, 1:453-461.

Justice, O. L. and Bass, L. N. 1978. In Principles and practices of seed storage, Agriculture Handbook No. 506, United States Department of Agriculture. pp:78-162.

Poonam, Dubey, R. K., Kumar, R. 2002. Effect of storage materials on seed germination and seed viability in Zinnia. Journal of Ornamental Horticulture, 5(2):59.

Rao, C. C., Dadlani, N. K. and Dadlani, M. 2003. Maintenance and enhancement of germination and vigour in marigold (Tagetes spp.) seed. Seed Science and Technology, 31(3):745-751.

Sang, C. K., Choi, B. J. and Koh, J. C. 1996. Effect of storage and temperature on seed germination of pasque flower
(Pulsatilla cernua var. koreana). Journal of the Korean Society for Horticultural Science, 37(3):447-450.

Selvaraju, P., Jacqueline, A. and Selvaraj. 1999. Effect of seed treatments and storage containers on viability and vigour of seed in marigold. (Tagetes erecta L.). South Indian Horticulture, 47:364-366.

Shakuntala, N. M., Vasudevan, S. N., Patil, S. B., Doddagoudar, S. A., Mathad, R. C., Macha, S. I. and Vijaykumar, A. G. 2012. Organic biopriming on seed vigour inducing enzyme in paddy an alternative to inorganics. The Ecoscan, 56:227-232.

Singh, R. K., Jain, S. K. and Singh, A. K. 2004. Seed storage of African marigold (Tagetes erecta L.) for exsitu conservation. Seed Science and Technology, 32(2):503-509.

Tang, W. M., Na, L. N., and Gang, Z. Z. 2009. Effect of temperature and storage length on seed germination and the effects of light conditions on seedling establishment with respect to seed size in Liguralia virgaurea. Plant Species Biology, 24(2):120-126.

Yogeesha, H. S., Janakiram, T., Bhanuprakash, K. and Nair, L. B. 2004. Seed storage studies in China aster (Callistephus chinensis L.). Journal of Ornamental Horticulture, 7 (3-4):128-131.

Zhang, M., Maeda, Y., Furihata, Y., Nakamaru, Y. and Esashi, Y. 1994. A mechanism of seed deterioration in relation to the volatile compounds evolved by dry seed themselves. Seed Science Research, 4:49-56.

\section{How to cite this article:}

Vaidya, S. B., P. B. Sable and Patil, V. B. 2021. Effect of Storage Conditions on Seed Viability of China Aster [Callistephus chinensis (L.) Nees]. Int.J.Curr.Microbiol.App.Sci. 10(10): 385401. doi: https://doi.org/10.20546/ijcmas.2021.1010.047 